\title{
The 3D magnetotelluric inversion system with static shift correction
}

\author{
Kun Zhang, Jiayong Yan \\ MLR Key Laboratory of Metallogeny and Mineral Assessment \\ Institute of Mineral Resources Chinese Academy of Geological Sciences \\ Beijing, China \\ zhangkun1010@163.com
}

\begin{abstract}
Besides the field acquisition of magnetotelluric is nearly $3 D$ exploration level, the $3 D$ data process and inversion have shortcomings. But our 3D inversion system has effectively resolved the problems of static shift and inversion efficiency. The system contains static shift correction, initial model design and inversion functions. In addition, the magnetotelluric data of the Middle and Lower Reaches of Tangtze Metallogenic were used to verify the system and confirm that $3 D$ MT is an effective method for deep exploration.
\end{abstract}

Keywords-magnetotelluric; static shift; correction; 3D inversion; system

\section{INTRODUCTION}

This Magnetotelluric (MT) is a geophysics exploration method for studying the crust and mantle electrical structure, which measures the surface orthogonal electric and magnetic fields base on the electromagnetic induction and natural electromagnetic fields. The static shift effect is a very common phenomenon in MT field data, which seriously impacts on the accuracy of data, and hence the interpretation of this method. There are five main static shift correction approaches, but they are all based on 1-D or 2-D MT theories and assumptions and have the uncertainty for correction results. And the $2 \mathrm{D}$ assumptions valid in some cases of interpretation, so 3D inversion methods has been the focus of the electromagnetic inversion method recently. But the computation efficiency is biggest problem for the inversion methods.

Base on the previous studies on the static shift correction and 3D inversion algorithms, we propose a new static shift correction method and improve the NLCG 3D inversion method, then combine them into the 3D MT inversion system..

\section{STATIC SHIFT CORRECTION AND VERIFICATION}

The static shift correction method is based on the 3D theory and real data. The static shift can be detected by the quantitative analysis of apparent parameters (apparent resistivity and impedance phase) of MT in high frequency range, and completed correction with inversion. The method is an automatic processing technology of computer with 0 cost, and avoids the additional field work and indoor processing with good results shown in Fig. 1.

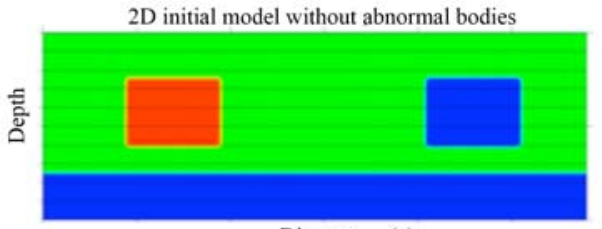

Distance (a)

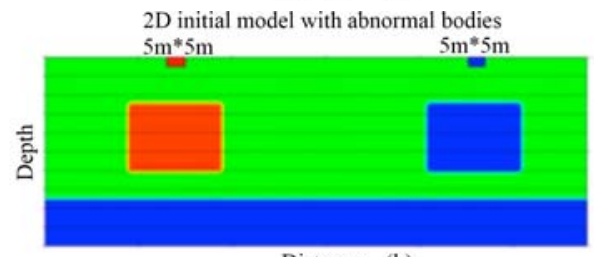

Distance (b)

2D inversion model (data without static shift)

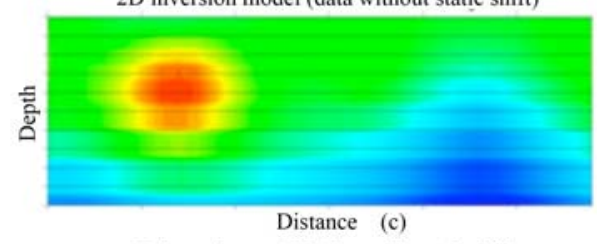

2D inversion model (data with static shift)

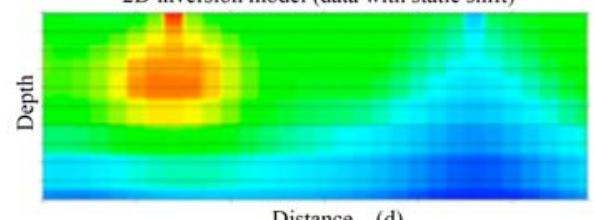

Distance (d)

2D inversion (data with static shift and corrected by the method)

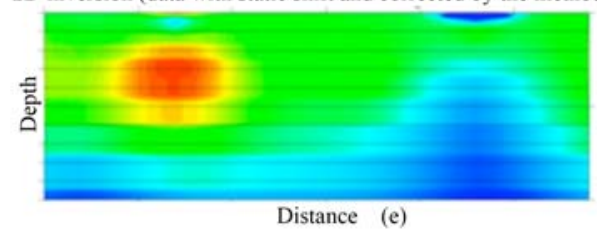

Fig. 1. Synthetic example. a: regular model; b: static shift model; c: inversion model using regular data; d: inversion model using static shift data without correction; e: inversion model using static shift data with correction

Fig. 1a shows a normal model (I) without any local heterogeneity. Fig. 1b shows a static-shifted model (II) with two local heterogeneous bodies (10 and $1000 \Omega \mathrm{m}$ ). Fig. 1c is the inversion result (A) for the synthetic data generated from model I. Fig. 1d is the inversion result (B) for the static-shifted data generated from model II. Fig. 1e is the inversion result (C) for the static-shifted data from model II, but with static shift 
correction. Inversion was calculated using the 2D NLCG method proposed by Rodi and Mackie ${ }^{[1]}$ with the same parameters $^{[1]}$.

As shown in Fig. 1d and e, the low- and high-resistivity channel observed in model B cannot be found in model $\mathrm{C}$. The resistivity value and the scale of the target bodies in model $\mathrm{C}$ are consistent with model $\mathrm{A}$. This confirms that the correction method is effective.

\section{3D INVERSION AND VERIFICATION}

The 3D inversion algorithm is improved based on the NLCG method of Newman \& Alumbaugh and Rodi \& Mackie $(2001)^{[1-3]}$. For the algorithm, we added the parallel structure, improved the computational efficiency, reduced the memory of computer and added the topographic factor. So the 3D inversion could work in general PC with high efficiency and accuracy. The application example of real data in the Middle and Lower Reaches of Tangtze Metallogenic is shown in Fig. 2.

The 3D electrical resistivity model for the test area is shown in Fig. 2c and d. In the slices shown, the similarities as well as the differences in the model are described and interpreted in the following. The most prominent feature revealed by the inversion was an extensive lower crust conductor (deep orange-red colors in Fig. 2b-d) located beneath the southern Ning-Wu Basin and sur- rounding areas (i.e., west of the Chaohu Fold Belt and north of the Fanchang Basin). The top of the feature is $10-20 \mathrm{~km}$ deep, and it possibly extends northward as far as the Ning-Wu Basin and southward to the Tongling Orogenic Belt. Its extent and other features are consistent with previous MT results presented by Chen and Zhang and Zhang et al. ${ }^{[4]}$ but the top depth is different, being found in this study to lie between the depths suggested in those earlier publications ${ }^{[4-5]}$.

The conductor has different features either side of the Chaohu Fold Belt. The vertical extent of the conductor west of the fold belt is greater than on the east, and the upper mantle on the eastern side has a higher resistance than on the western side. The conductor has steep boundary interfaces in Zone I (Fig. 2) and tends to be shallower in the SE, with a top depth of about $12-15 \mathrm{~km}$ and high resistance material beneath it. The bottom interface depth of the feature coincides with the depth of the Moho (28-32km) as deduced from seismic and geological information. Then we discuss the lower crust 'liquid', the upper mantle uplift, the faults and so on base on the 3D inversion model of MT data.

\section{CONCLUSIONS}

We construct a system for static shift correction and 3D inversion of MT and make the 3D inversion with static shift correction work on PC instead of large server. The real data are used to verify the algorithms. And the application results shows that the 3D MT exploration (with 3D inversion) is an effective method for the study of deep structure.
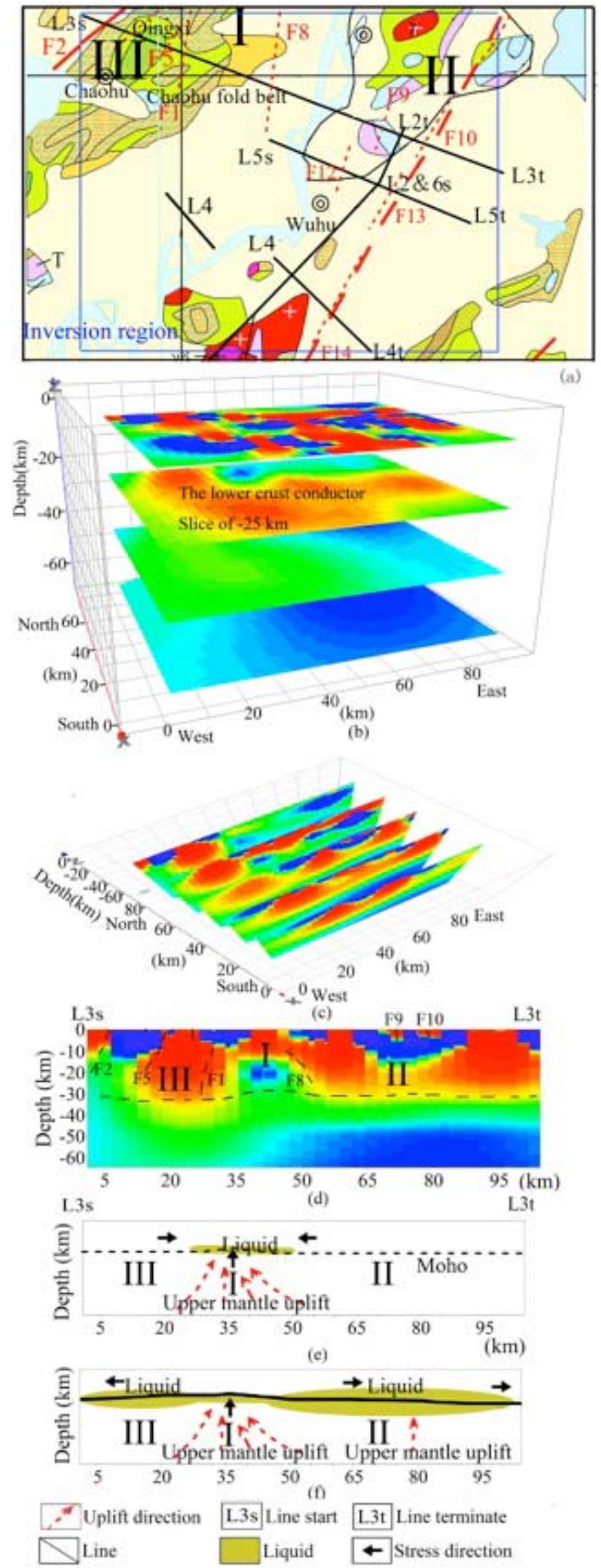

Fig. 2. 3D MT inversion model and interpretation inversion model

\section{ACKNOWLEDGMENT}

The study is supported by the geological survey project" Integration of prospecting and exploration technology for deep ore prospecting of fractured altered rock type gold deposit” and the help of work group. 


\section{REFERENCES}

[1] W.L. Rodi, R.L. Mackie, "Nonlinear conjugate gradients algorithmfor 2 D magnetotelluric inversion,”. Geophysics, vol. 66, pp. 174-187, January 2001.

[2] G.A. Newman, M. Hoversten, E. Gasperikova, P.E. Wannamaker, “3-D Magnetotelluric Characterization of the Coso Geothermal Field," Thertieth Workshop on Geothermal Reservoir Engineering, Stanford University, California, 2005, pp.2.

[3] K. Zhang, H. Dong, J.Y. Yan, Q.T. Lu , W.B. Wei, Y.X. He, “A NLCG inversion method of magnetotellurics with parallel structure,” Chinese journal of geophysics, vol. 56, pp. 3922-3931, November 2013 (in Chinese).

[4] K. Zhang, W.B. Wei, Q.T. Lu, H. Dong, Y.Q. Li, “Theoretical assessment of 3-D magnetotelluric method for oil and gas exploration: Synthetic examples,” Journal of Applied Geophysics, Vol. 106, pp. 23-36, Aperal 2014.

[5] K. Zhang, Q.T. Lu, J.Y. Yan, "The lower crust conductor from Nanjing(Ning)-Wuhu(Wu)area in the Middle and Lower Reaches of Yangtze River: Preliminary results from 3D inversion of magnetotelluric data,” Journal of Asian Earth Sciences, Vol. 101, pp. 20-29, June 2015. 